\title{
Image quality and radiation exposure with low-contrast-dose computed tomography angiography of the lower extremities
}

\author{
Abed Al Nasser Assi \\ Department of Medical Imaging, Arab American University, Jenin, Palestine \\ Department of Medical Imaging, An-Najah National University, Nablus, Palestine
}

\section{Abstract}

Introduction: Multidetector computed tomography is the reference standard for the diagnosis of peripheral arterial disease (PAD). The aim of this study is to optimise the image quality of computed tomography (CT) scanning for the diagnosis of PAD with the lowest possible radiation and contrast volume.

Material and methods: Seventy-two patients were referred for evaluation of suspected PAD with CT angiography. Patients were randomly assigned to an optimise care dose of $\mathrm{kVp}$ - group A, $n=36 ; 18$ men, 18 women; mean age, 63 years with standard deviation 15; range, $20-88$ years (contrast volume $80-85 \mathrm{ml}$ was injected automatically with bolus tracking, and group B, $n=36 ; 18$ men, 18 women; mean age, 61 years with standard deviation 16; range, 26-88 years (contrast volume $120-140 \mathrm{ml}$ was injected automatically with bolus tracking). Other scanning parameters were kept constant. Lower extremities vessel enhancement and image noise were quantified, and the signal-to-noise ratio (SNR) and contrast-to-noise ratio (CNR) were calculated. Subjective vessel contrast was assessed by two radiologists in consensus.

Result: A total of 16 cases of PAD (22.2\%) were found in the evaluated of subjects (10 in group A, and six in group B). All PAD cases were detected by the two readers. There was no significant difference in the size or location of the PAD between the two groups; the average image noise was $19 \mathrm{HU}$ for group A and $16 \mathrm{HU}$ for group B. The difference was not statistically significant $(p=0.183)$. Overall, the SNR and CNR were slightly higher in group B (25.5 and 24.1, respectively) compared with group A (20 and 17.4, respectively), but those differences were not statistically significant ( $p=061$ and $p=0.38$, respectively).

Conclusions: All patients were evaluated by lower extremities CTA protocol allowing similar image quality to be achieved in both groups, with optimised care dose for both protocols, and contrast volume was reduced by $40 \%$ in the new protocol group compared to the conventional protocol group.

Key words: peripheral arterial disease, lower extremities, signal to noise ratio, contrast to noise ratio, contrast volume, image quality.

\section{Introduction}

Multidetector computed tomography (MDCT) is the reference standard for the diagnosis of peripheral arterial disease $(\mathrm{PAD})[1,2]$. It is reported that PAD is the second most common disease, after myocardial infarction and stroke, and results in an estimated [3]. Early diagnosis and appropriate medical interventions can mitigate limb- specific symptoms, improve quality of life, and decrease systemic cardiovascular risk [4]. Computed tomography angiography (CTA), as a non-invasive and safe examination, has been widely used in clinical practice to diagnose $\mathrm{PAD}$ and acute lower-extremity vascular injury with high accuracy [5-8].

Several studies had revealed the overuse of CTA to exclude PAD, which increases in effective dose per patient [9],

\section{Correspondence address:}

Dr. Abed Al Nasser Assi, Arab American University, Jenin, Palestine, e-mail: Abed.Assi@aaup.edu

Authors' contribution:

A Study design · B Data collection · C Statistical analysis · D Data interpretation · E Manuscript preparation · F Literature search · G Funds collection 
so there is a need for reduction of radiation dose. Therefore, the recent studies of PAD CTA have conducted important updates on optimising protocols that restrict the amount of iodinated contrast media, thus decreasing the risk of contrast medium-induced nephropathy, particularly in people suffering from certain diseases such as diabetes and high blood pressure [10]. Thus, lowering the radiation dose by using $128 \mathrm{MDCT}$ scanning and low contrast medium volume CTA protocols is potentially advantageous both to reduce radiation-derived risks and to prevent contrast medium-induced adverse events.

The combination of $70 \mathrm{kVp}$ and high-pitch acquisition technique holds the potential to further reduce the radiation dose with a reduction of the amount of iodine contrast injected during the image scan. The aim of our study was therefore to investigate the feasibility, image quality, and radiation dose of lower extremity CTA using $70 \mathrm{kVp}$ combined with high pitch acquisition and less iodinated contrast medium injected during PAD scanning.

\section{Material and methods}

\section{Subjects}

This study was held by An-Najah National University in Nablus city, Palestine. Each individual was referred for lower extremity CTA for clinical suspicion of PAD exam. To be eligible for the study patients had to have normal renal function. Patients with a personal history of allergy to iodinated contrast material, or impaired renal function (creatinine $>1.2 \mathrm{mg} / \mathrm{dl}$ ) were excluded from the study. The study subjects were divided into two groups. Patients in group A (18 male and 18 female, mean age 63 years, standard deviation 15 , range $20-88$ years) underwent a novel lower extremity CTA protocol consisting of optimised care dose selection and low contrast medium volume ( $80-85 \mathrm{ml}$ ) acquisition, whereas group B (18 male and 18 female, mean age 61 years, standard deviation 16 , range 26-88 years) had conventional PAD CTA protocol with optimised care dose selection and iodine contrast volume (120-140 ml).

\section{Lower extremities computed tomography angiography protocol}

All lower extremities CTA examinations were performed using a 124MD CT system (Somatom Definition, Siemens Healthcare, Forchheim, Germany) for group A - the acquisition parameters were: pitch $=1.3$, low contrast medium volume $=80-85$; flow rate $=4$; bolus tracking technique with Hounsfield attenuate threshold $=80-85 \mathrm{HU}$, and delay scan time after the contrast triggering in bolustracking technique 6 seconds without breathing instruction before data acquisition. For group $B$ the acquisition parameters were: pitch $=0.8$; contrast $=120-140 \mathrm{ml}$; flow rate $=4 ; \mathrm{HU}$ predefined attenuation threshold with delay scan time after the contrast triggering in bolus-tracking technique 8 seconds with breathing instructions before data acquisition, bolus tracking with the region of interest placed in the main descending aorta at a level L2-L3 (care dose were selected for both group protocols), pure, undiluted iodinated contrast medium was used for both groups (Imoron 300), other parameters kept constant for both groups (slice thickness $3 \mathrm{~mm}, \mathrm{kVp}=70$, and care dose selected for both groups).

\section{Quantitative analysis of pulmonary computed tomography angiography images}

All images were evaluated by two observers with one and three years of experience in CTA evaluation, blinded to the clinical history and lower extremity CTA protocol. Each study was subjectively classified as diagnostic or non-diagnostic by each reader.

The signal intensity for the peripheral arteries (SI vessels), given in $\mathrm{HU}$, was defined as the attenuation measured by placing circular regions of interest (ROIs) in the centre of these vessels. The ROI size was adapted to the diameter of the vessel, reaching up to $40 \mathrm{~mm}^{2}$ in the central arteries. The main arteries were analysed by select common iliac, femoral, and popliteal artery left and right. In order to minimise bias, each artery was measured at three different locations. The mean of these values was used for further calculations.

The attenuation in the peripheral lower extremities arteries was ignored because unable to use the ROI method was not possible for assessment of the signal intensity due to their small calibre.

To define the image noise, the standard deviations of $\mathrm{HU}$ measured in ROIs of at least $40 \mathrm{~mm}^{2}$ drawn in three different regions outside the patient body (middle, left, and right sides) were averaged.

The signal-to-noise ratio (SNR) was defined as SI vessel/noise. The contrast-to-noise ratio (CNR) was defined as ([SI vessel - SI background]/noise). These two parameters were calculated separately for the central average for lower extremities arteries.

For statistical analysis SPSS software version 16.0 (SPSS Inc., Chicago, IL, USA) was used, and the independent sample $t$-test was employed to compare continuous variables. A two-sided $p$-value $<0.05$ was considered to indicate a statistically significant difference.

\section{Result}

Among 16 consecutive individuals with suspected PAD CTA, without complications there were no significant differences observed in age, weight, and gender between groups A and B. Patient data are presented in Table 1. All studies were considered to have enough image quality to detect PAD (Figure 1). A total of 16 cases of PAD (22.2\%) were found in the evaluated of subjects (10 in group A and 
six in group B). All PAD cases were detected by the two readers. There was no significant difference in the size or location of the PAD between the two groups.

The average image noise was $19 \mathrm{HU}$ for group $\mathrm{A}$ and $16 \mathrm{HU}$ for group B. The difference was not statistically significant $(p=0.183)$. Overall, the SNR and CNR were slightly higher in group B (25.5 and 24.1, respectively) compared with group A (20 and 17.4, respectively), but those differences were not statistically significant $(p=0.61$ and $p=0.38$, respectively).

\section{Discussion}

In the present study, the two groups were matched by gender, age, and weight, thus exclude possible variations between the groups in the vessel enhancement secondary to a different body habitus. Conversely, volume, concentration, injection rate, and duration of iodinated contrast medium administration are factors directly associated with lower extremities artery enhancement. As shown, the reduction in acquisition times achieved with the newest CT equipment avoids the need for long-duration contrast medium administration protocols because the injection duration critically affects both the magnitude and timing of contrast enhancement [11-20], and this study used a 128 MDCT Siemens machine during lower extremities CTA scanning and managed to reduce the injection period to less than $50 \%$ during $\mathrm{CT}$ scanning, and this in turn reduced the size of the contrast medium to less than $40 \%$ of the value used in the classical lower extremities CTA scanning protocol.

The most relevant finding of this study is that in individuals weighing on average $85 \mathrm{~kg}$, a lower extremities CTA protocol with relatively small volume of iodinated contrast medium $(80 \mathrm{ml})$ results in lower extremities CT angiograms with lower injection period than when using a conventional lower extremities CTA protocol (Figure 1). The novel protocol proposed here yielded a diagnostic confidence of lower extremities that was not significantly different from the standard protocol but using $40 \%$ less intravenous contrast medium. This optimisation of a standard lower extremities CTA protocol may have been achievable due to the latest technology, such as thin collimation and shortened study time provided by the new MDCT equipment, the use of automatic initiation of the examination by the bolus tracking technique, and the infusion of a saline flush immediately following the contrast medium, which increase peak aortic enhancement by pushing contrast material into the cardiovascular system, which otherwise would be left in the injection tubing. Also the saline flush minimises streak artefacts from dense contrast, which increases SNR of the lower extremities [12,13].

It is known both that formal and CT lower extremity angiographies involve the injection of intravenous radiographic contrast under pressure, and this injection may develop cardiopulmonary arrest after contrast in-
Table 1. Structure of population study data, patient characteristics, signal intensity for the central pulmonary arteries, and image quality in the study groups

\begin{tabular}{|l|c|c|c|}
\hline Parameter & Group A & Group B & $p$ value \\
\hline Male, $n$ & 18.0 & 18.0 & \\
\hline Female, $n$ & 18.0 & 18.0 & \\
\hline Weight, $\mathrm{kg}$ & 83.4 & 82.6 & 0.92 \\
\hline Vessel (attenuation), HU & 289.0 & 291.0 & 0.81 \\
\hline Noise & 16.0 & 19.0 & 0.18 \\
\hline Signal-to-noise ratio (SNR) & 20.0 & 25.4 & 0.62 \\
\hline Contrast-to-noise ratio (CNR) & 17.4 & 24.1 & 0.38 \\
\hline
\end{tabular}
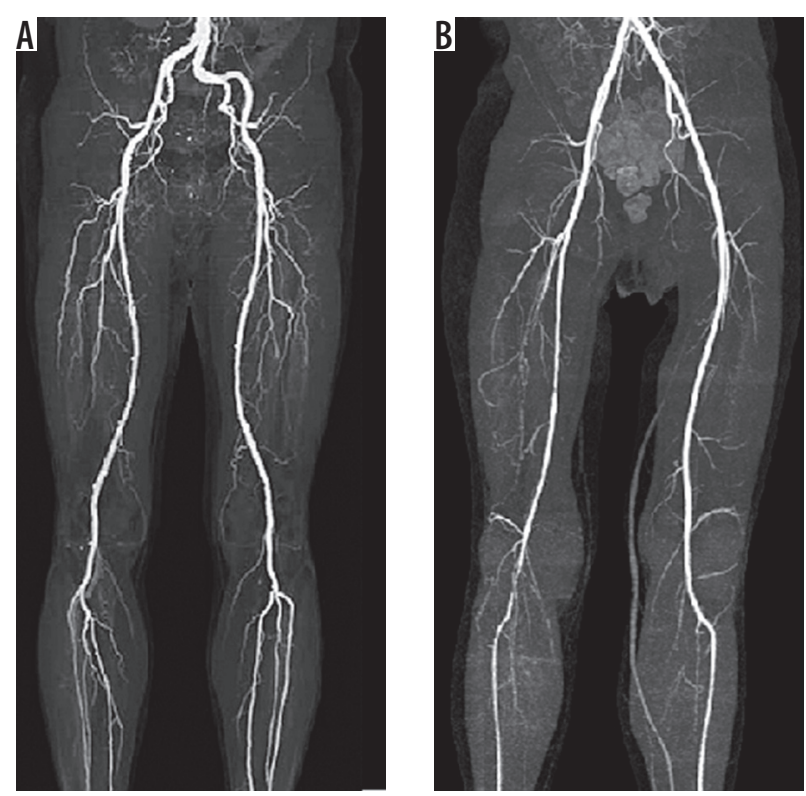

Figure 1. Exemplary $3 \mathrm{~mm}$ transverse peripheral arterial disease (PDA) computed tomography angiography images at $70 \mathrm{kVp}$ from the present patient collective. A) Image scan with protocol A (contrast volume 80-85 cc and pitch $=1.3$ ). B) Image scan with protocol B (contrast volume $120-140 \mathrm{cc}$ and pitch $=0.8$ )

jection $[21,22]$, so it is thought. The reduced amount of contrast to $40 \%$ undoubtedly will reduce the likelihood of cardiac arrest, and in turn will improve the use of CTA in diagnostic lower extremities in a large scale. Also it is noticeable that when the amount of contrast injection is reduced, it reduces beam hardening and streak artefacts (see Figure 1) causing obscuring adjust vessels during image scans, especially in the area of the superior vena cava.

The main limitations in this study are that it was a retrospective study analysis. In order to spare participants from double dose exposure, the study used data existing on the database in the hospital that were used in the survey of previously unknown lower extremities scanning and then compared them with the new protocols that use new standards through the screening to reduce the size of the contrast medium combined with a high injection rate, and this may strain the final results. Hence, in order to 
avoid any bias during comparison between the two scanning protocols, the author recommends that the data was collected from the same subjects on both protocols, and that iodinated contrast agents are needed for helical CT PAD angiography, and their use may not be possible in patients with impaired renal function or a severe allergy to the contrast material.

The present study demonstrates the feasibility of lower extremity CTA using $70 \mathrm{kVp}$ combined with high pitch acquisition with a selective care dose option during image scanning, so there is no significant difference in radiation dose between the two protocols but a decrease of about $40 \%$ in the volume of contrast material compared to the standard protocol used in An-Najah hospital medical imaging department without compromising image quality. Lowering the tube voltage is an effective method to reduce radiation dose because of the exponential relationship between voltage and radiation dose [13]. Lowering tube voltage from $120 \mathrm{kVp}$ to $70 \mathrm{kVp}$ has been reported to result in a $30 \%$ reduction in radiation dose for lower extremity CTA [22]. In addition, increasing pitch is another method to reduce radiation dose by shortening the image acquisition time [14]. Amacker et al. reported that the mean radiation dose decreased by $20 \%$ and $42 \%$ when the pitch was increased from 1 to 3.2 for routine chest and abdominal CT scans, respectively [15], but it in our case there was no decrease in the amount of the dose due to the care dose option being selected during the image scan; this option increases the mAs during image scanning to reduce image noise, which led to increased image quality. On other hand, a decrease in the $\mathrm{kVp}$ during CTA scan will increased the attenuation of each unit of low iodine concentration because the low voltage helps improve blood vessels and elasticity while minimising the absorption of radiation at the same time, as long as there is no significant scattering of the beam or degradation of the image due to large tissue or bone along the X-ray path [6]. The reduction of the amount of contrast media also leads to the reduction of the cost and risk of side effects resulting from giving iodine in large quantities.

\section{Conflict of interest}

The authors declare that they have no conflict of interest.

\section{References}

1. Horehledova B, Mihl C, Milanese G, et al. CT angiography in the lower extremity peripheral artery disease feasibility of an ultra-low volume contrast media protocol. Cardiovasc Interv Radiol 2018; 41: 1751-1764.

2. Schäfer JC, Haubenreisser H, Meyer M, et al. Feasibility of a single contrast bolus high-pitch pulmonary CT angiography protocol followed by low-dose retrospectively ECG-gated cardiac CT in patients with suspected pulmonary embolism. Fortschr Röntgenstr 2018; 190: 542-550.

3. Foley WD, Stonely T. CT angiography of the lower extremities. Radiol Clin North Am 2010; 48: 367-396.

4. Hirsch AT, Criqui MH, Treat-Jacobson D, et al. Peripheral arterial disease detection, awareness, and treatment in primary care. JAMA 2001; 286: 1317-1324.

5. Schreiner MM, Platzgummer H, Unterhumer S, et al. A BMIadjusted ultra-low-dose CT angiography protocol for the peripheral arteries-image quality, diagnostic accuracy and radiation exposure. Eur J Radiol 2017; 93: 149-156.

6. Buls N, de Brucker Y, Aerden D, et al. Improving the diagnosis of peripheral arterial disease in below-the-knee arteries by adding time-resolved CT scan series to conventional run-off CT angiography. First experience with a 256-slice CT scanner. Eur J Radiol 2019; 110: 136-141.

7. Klink T, Wilhelm T, Roth C, Heverhagen JT. Dual-energy CTA in patients with symptomatic peripheral arterial occlusive disease: study of diagnostic accuracy and impeding factors. Fortschr Röntgenstr 2017; 189: 441-452.

8. Mishra A, Jain N, Bhagwat A. CT angiography of peripheral arterial disease by 256-slice scanner: accuracy, advantages and disadvantages compared to digital subtraction angiography. Vasc Endovascular Surg 2017; 51: 247-254.
9. Liu B, Gao S, Chang Z, Wang C, Liu Z, Zheng J. Lower extremity CT angiography at $80 \mathrm{kVp}$ using iterative model reconstruction. Diagn Interv Imaging 2018; 99: 561-568.

10. Kim JW, Choo KS, Jeon UB, et al. Diagnostic performance and radiation dose of lower extremity $\mathrm{CT}$ angiography using a 128-slice dual source CT at $80 \mathrm{kVp}$ and high pitch. Acta Radiol 2016; 57: 822-828.

11. Bae KT. Peak contrast enhancement in CT and MR angiography: when does it occur and why? Pharmacokinetic study in a porcine model. Radiology 2003; 227: 809-816.

12. Han JK, Kim AY, Lee KY, et al. Factors influencing vascular and hepatic enhancement at CT: experimental study on injection protocol using a canine model. J Comput Assist Tomogr 2000; 24: 400-406.

13. Schoellnast H, Deutschmann HA, Berghold A, Fritz GA, Schaffler GJ, Tillich M. MDCT angiography of the pulmonary arteries: influence of body weight, body mass index, and scan length on arterial enhancement at different iodine flow rates. AJR Am J Roentgenol 2006; 187: 1074-1078.

14. Al Nasser Assi A, Abu Arra A. Optimization of image quality in pulmonary CT angiography with low dose of contrast material. Polish Journal of Medical Physics and Engineering 2017; 23: 43-46.

15. Schicchi N, Fogante M, Oliva M, et al. Radiation dose and image quality with new protocol in lower extremity computed tomography angiography. Radiol Med 2019; 124: 184-190.

16. Schreiner MM, Platzgummer H, Unterhumer S, et al. Multipath curved planar reformations of peripheral CT angiography: diagnostic accuracy and time efficiency. Cardiovasc Interventional Radiol 2018; 41: 718-725.

17. Beeres M, Juhee K, Bucher AM, et al. Automated attenuation based tube potential selection of the lower extremity runoff: a comparison 
to fixed kilovolt with automated tube current modulation. J Comput Assist Tomogr 2017; 41: 817-822.

18. Masuda T, Funama Y, Nakaura T, et al. CT angiography of suspected peripheral artery disease: comparison of contrast enhancement in the lower extremities of patients undergoing and those not undergoing hemodialysis. AJR Am J Roentgenol 2017; 208: 1127-1133.

19. Bae KT, Heiken JP. Scan and contrast administration principles of MDCT. Eur Radiol 2005; 15 (Suppl 5): E46-E59.

20. Erturk SM, Ichikawa T, Sou H, Tsukamoto T, Motosugi U, Araki T. Effect of duration of contrast material injection on peak enhance- ment times and values of the aorta, main portal vein, and liver at dynamic MDCT with the dose of contrast medium tailored to patient weight. Clin Radiol 2008; 63: 263-271.

21. Higashigaito K, Schmid T, Puippe G, et al. CT angiography of the aorta: prospective evaluation of individualized low-volume contrast media protocols. Radiology 2016; 280: 960-968.

22. Rubin GD, Schmidt AJ, Logan LJ, et al. Multi-detector row CT angiography of lower extremity arterial inflow and runoff: initial experience. Radiology 2001; 221: 146-158. 